gr-qc/9306024 Alberta-Thy-30-93 May '93

\title{
Faraday lines and observables for the Einstein-Maxwell theory
}

\author{
Viqar Husain \\ Theoretical Physics Institute, University of Alberta, \\ Edmonton, Alberta, Canada T6G 2J1
}

\begin{abstract}
In recent work on Einstein gravity in four dimensions using the Ashtekar variables, non-local loop variables have played an important role in attempts to formulate a quantum theory. The introduction of such variables is guided by gauge invariance, and here an infinite set of loop variables is introduced for the Hamiltonian form of the Einstein-Maxwell theory. The loops that enter the description naturally are the (source free) electric field lines. These variables are invariant under spatial diffeomorphisms and they also form a closed Poisson algebra. As such they may be useful for quantization attempts and for studying classical solutions.
\end{abstract}

PACS numbers: 4.20.-q, 4.20.Fy, 4.20.Me, 4.60.+n

Typeset using REVTEX 
The canonical approach to quantum gravity in four dimensions has received much attention in recent years due to the introduction by Ashtekar of new Hamiltonian variables for general relativity [1]. These phase space variables, an electric field and vector potential, are similar to those for $\mathrm{SO}(3)$ Yang-Mills theory, and so allow the introduction of holonomies or Wilson loops as natural variables on the phase space.

Classical phase space variables based on loops have been of substantial use in canonical quantum gravity. Rovelli and Smolin introduced an infinite set of gauge invariant loop variables on the Ashtekar phase space which are made from holonomies of the vector potential traced together with the conjugate electric field. These variables form a closed Poisson algebra, and a representation of this algebra on functions of loops has been used to study the quantum theory [2]. The Hamiltonian and spatial diffeomorphism constraints of general relativity may be written as operators on this loop space [2,3]. The main result of this work is that there are an infinite set of solutions of the Dirac quantization conditions in the loop representation, and the physical states so obtained are labelled by knot invariants [2]. This labelling arises essentially because the diffeomorphism invariant information in a loop is its knot class.

Many open problems remain in this approach, and among them is the question of what classical variables are to be represented as Hermitian operators on the space of physical states. The natural answer to this is that the variables should be invariant under all the gauge symmetries of the theory. However requiring this for general relativity amounts to finding constants of the motion, since the Hamiltonian constraint generates both gauge and dynamics. In particular, a complete set of fully gauge invariant variables amounts to complete integrability of the full Einstein equations. More practical may be a weaker condition, namely requiring gauge invariance under only the kinematical gauge symmetries - spatial diffeomorphisms and Yang-Mills symmetries. The classical and quantum evolution of these variables via the Hamiltonian constraint can then be studied.

The Rovelli-Smolin loop variables mentioned above are not invariant under spatial diffeomorphisms. In pure gravity it isn't possible to construct loop variables that are spatially 
diffeomorphism invariant, essentially because loops are external to the phase space, and phase space functionals that depend on external variables cannot be made diffeomorphism invariant. On the other hand loop variables seem essential to constructing a loop representation, and it is only in this representation that a large number of solutions of the Dirac quantization conditions have been obtained. It is therefore natural to ask if there is a way of obtaining diffeomorphism invariant loop observables when there is coupling to matter fields. This is important also from the point of view of incorporating matter into the loop space formalism, and the idea presented below may provide a way of doing this.

In this letter the question of gauge invariant variables is discussed when there is coupling of Einstein gravity to the Maxwell field. First the Einstein-Maxwell Hamiltonian system is given in the Ashtekar variables and the Maxwell Gauss law is solved classically to obtain a partially reduced system. Phase space loop variables that are invariant under the gravity Gauss law and spatial diffeomorphism constraints are then presented. This is the main result of the paper. This is followed by a discussion of the possible uses of these variables.

The phase space variables for the Einstein-Maxwell system may be taken as the Ashtekar variables $\left(A_{a}^{i}, E^{a i}\right)$ for the gravitational field, and the pairs $\left(a_{a}, e^{a}\right)$ for the Maxwell field. $a, b, c \ldots$ are spatial indices and $i, j, \ldots$ are the internal $\mathrm{SO}(3)$ indices. The phase space constraints reflecting the symmetries are

$$
\begin{aligned}
& \partial_{a} e^{a}=0, \\
& D_{a} E^{a i}=0, \\
& F_{a b}^{i} E^{b i}+f_{a b} e^{b}=0, \\
& \epsilon^{i j k} F_{a b}^{k} E^{a i} E^{b j}+(\operatorname{det} E)^{-2} E^{a i} E^{c i} E^{b j} E^{d j}\left(e_{a b} e_{c d}+f_{a b} f_{c d}\right)=0 .
\end{aligned}
$$

where $D_{a}$ is the covariant derivative of $A_{a}^{i}$ and $F_{a b}^{i}$ is its curvature, $\operatorname{det} E$ is the determinant of the inverse of $E^{a i}, e_{a b}=\epsilon_{a b c} e^{c}$, and $f_{a b}=\partial_{[a} a_{b]}$. The first two constraints are the Maxwell and gravity Gauss law constraints, the third is the spatial diffeomorphism constraint, and the last is the Hamiltonian constraint. The total Hamiltonian $H$ is a linear combination of these constraints and the phase space variables evolve via the Hamilton equations. 
Starting with this constrained system, the goal is to first extract at the classical level the two local unconstrained degrees of freedom of the Maxwell field, and then eliminate $\left(a_{a}, e^{a}\right)$ in favor of these in the constraints. This partially reduced system of constraints will then be used as the starting point for finding the gauge invariant observables.

The reduction may be done by finding a solution of the Hamilton-Jacobi equation associated with the Maxwell Gauss law constraint [4], which is obtained by the replacement $e^{a} \rightarrow \delta S / \delta a_{a}$ in (1). The solution must have two integration variables $u_{n}(n=1,2)$ corresponding to the two degrees of freedom. A solution is

$$
S\left[a ; u_{1}, u_{2}\right]=\int d^{3} x \epsilon^{a b c} a_{a} \partial_{b} u_{1} \partial_{c} u_{2}
$$

The electric field $e^{a}$ is then given by

$$
e^{a}=\frac{\delta S}{\delta a_{a}}=\epsilon^{a b c} \partial_{b} u_{1} \partial_{c} u_{2}
$$

which solves the Maxwell Gauss law. The momenta conjugate to the reduced variables $u_{n}$ are given in the usual way from the Hamilton-Jacobi functional:

$$
\begin{aligned}
& p_{u_{1}}=\frac{\delta S}{\delta u_{1}}=\frac{1}{2} \epsilon^{a b c} f_{a b} \partial_{c} u_{2} \\
& p_{u_{2}}=\frac{\delta S}{\delta u_{2}}=-\frac{1}{2} \epsilon^{a b c} f_{a b} \partial_{c} u_{1}
\end{aligned}
$$

These relations may be used to eliminate the six local $\left(a_{a}, e^{a}\right)$ variables for the four reduced ones $\left(u_{n}, p_{u_{n}}\right)$ in the constraints $(3)$ and (4). This gives the reduced system. In particular the diffeomorphism constraint (3) reduces to

$$
C_{a} \equiv F_{a b}^{i} E^{b i}+p_{u_{n}} \partial_{a} u_{n}=0
$$

The aim now is to find a set of observables $T$ that are invariant under the transformations generated by the gravity Gauss law (2) and the reduced spatial diffeomorphism constraints (9). By definition, the $T$ must have weakly vanishing Poisson brackets with these kinematical constraints.

The configurations of the Maxwell fields $u_{1}=c_{1}$ and $u_{2}=c_{2}$ for constants $c_{1}, c_{2}$ define two surfaces, and their intersection gives a loop. Since $e^{a}=\epsilon^{a b c} \partial_{b} u_{1} \partial_{c} u_{2}$, the electric field 
lines are in fact parallel to these loops. The idea now is to define the gravity loop observables based on these electric field loops $\gamma\left[u_{n}\right]\left(c_{1}, c_{2}\right)$ rather than auxiliary loops. Such a construction will, if done properly, make all the gravity loop variables [2] invariant under spatial diffeomorphisms as well.

The first loop observable is the trace of the holonomy, and with the loops chosen as above it becomes diffeomorphism invariant:

$$
T^{0}\left[A, u_{n}\right]\left(c_{1}, c_{2}\right)=\operatorname{Tr} U \equiv \operatorname{TrPexp} \int_{\gamma\left[u_{n}\right]} d x^{a} A_{a}
$$

The next two observables have one and two insertions of the triad $E^{a i}$ in the holonomies and are defined by

$$
\begin{aligned}
T^{1}\left[A, E, u_{n}\right]\left(c_{1}, c_{2}\right)= & \int_{\gamma\left[u_{n}\right]} d s w_{a}(s) \operatorname{Tr}\left[E^{a}(\gamma(s)) U_{\gamma}(s, s)\right] \\
T^{2}\left[A, E, u_{n}\right]\left(c_{1}, c_{2}\right)= & \int_{\gamma\left[u_{n}\right]} d s \int_{\gamma\left[u_{n}\right]} d t w_{a}(s) w_{b}(t) \\
& \times \operatorname{Tr}\left[E^{a}(\gamma(s)) U_{\gamma}(s, t) E^{b}(\gamma(t)) U_{\gamma}(t, s)\right],
\end{aligned}
$$

where the 1-form density

$$
w_{a} \equiv \epsilon_{a b c} \dot{\gamma}^{b} \frac{\delta \gamma^{c}}{\delta u_{1}}
$$

$\dot{\gamma}^{a}=d x^{a} / d s$ is the tangent vector to the loop, $s$ is a parameter along the loop, and $U(s, t)$ is the holonomy between the parameter values $s, t$. In (13), $u_{1}$ may be replaced by $u_{2}$ to give distinct observables. For comparison, the traces in the integrands of (11) and (12) are the first two Rovelli-Smolin loop variables [2].

The generalizations $T^{N}$ of these for $N$ triad insertions are obtained in a similar way. These observables are functionals of $A_{a}^{i}, E^{a i}, u_{n}$, and functions of the two parameters $c_{1}, c_{2}$, and are independent of the momenta $p_{u_{n}}$ conjugate to the $u_{n}$. The Poisson brackets $\left\{T^{M}, T^{N}\right\}$ are therefore determined solely by the gravitational variables, and are non-zero for any two $T^{N}$ only if the corresponding loops intersect. More generally, the loops may be determined by $f\left(u_{1}, u_{2}\right)=c_{1}$ and $g\left(u_{1}, u_{2}\right)=c_{2}$ for arbitrary functions $f, g$. So given a 
configuration of the phase space variables and two arbitrary functions $f, g$, the loop variables will in general not commute and the Poisson algebra will be of the form

$$
\left\{T^{M}, T^{N}\right\} \sim T^{M+N-1}
$$

This is of the same form as that of the gravity loop variables with auxiliary loops [2].

Since the $T^{N}$ described above do not depend on all the phase space variables, they cannot form a complete set of observables. Observables depending on the momenta are $P_{n}=\int d^{3} x p_{u_{n}}$ but with these, the Poisson algebra no longer retains the nice form (14). An infinite number of new observables are generated from the $T^{N}$ by Poisson brackets with $P_{n}$, since these act to shift the loops and introduce additional derivatives on the 1-form densities $w_{a}$. The algebra can of course be completed by exhausting all possible Poisson brackets.

For the quantum theory, the Dirac quantization conditions in the configuration representation are obtained by the replacements $E^{a i} \rightarrow \delta / \delta A_{a}^{i}$ and $p_{u_{n}} \rightarrow \delta / \delta u_{n}$ in the constraints. Then the wavefunctional

$$
\Psi\left[A, f\left(u_{1}, u_{2}\right), g\left(u_{1}, u_{2}\right)\right]\left(c_{1}, c_{2}\right)=\operatorname{TrPexp} \int_{\gamma[f, g]} d x^{a} A_{a}
$$

solves the diffeomorphism and Gauss law conditions. This solution is parametrized by two arbitrary functions $f$ and $g$, and the constants $c_{1}, c_{2}$. Furthermore it is also annihilated by the gravitational part of the Hamiltonian constraint. The full action of the Hamiltonian constraint (4) is complicated by the determinant terms in the Maxwell part. This latter term is in fact non-local when the inversions of (7) and (8) are substituted in (4).

For the quantum theory in the loop representation, the $T^{N}$, together with all the observables required to complete them, provide a natural set of observables to represent as operators on the solution space of the constraints. These must commute with the diffeomorphism operator and have the appropriate evolution with respect to the Hamiltonian constraint operator.

The basic idea that is utilized above to construct diffeomorphism invariant observables is the use of matter fields to define loops. This is related to the general idea of using 
matter reference systems for gravitational variables, and it has a long history. It is discussed for a point particle by DeWitt [5], and more recent discussions in related contexts have been by Rovelli [6], and by Kuchar and Torre [7]. The latter have used particular types of matter which is defined by certain coordinate conditions. Their approach fixes all the coordinates by reference to matter fields, whereas here only the loops have been fixed by matter configurations. A similar approach to loop variables for couplings to scalar fields has also been discussed by the author [8]. In the context of the Ashtekar variables, matter fields have been used to construct surface observables using scalar fields [9], and antisymmetric tensor fields [10]. These surface observables are well defined in terms of loop observables, and they exibit a discrete spectrum in the loop representation, with the areas quantized in units of the Planck area [1].

In summary, an infinite set of diffeomorphism invariant loop observables for the Einstein-Maxwell system have been described. The construction used electric field lines as the reference systems for the gravitational loop variables. It is clearly possible to construct loop observables of this kind using any matter fields. 


\section{REFERENCES}

[1] A. Ashtekar, Non-perturbative canonical gravity, Lecture notes in collaboration with R. S. Tate

(World Scientific, Singapore, 1991).

[2] C. Rovelli and L. Smolin, Phys. Rev. Lett. 61, 1155

(1988); Nucl. Phys. B331, 80 (1990).

[3] M. Blencowe, Nucl. Phys. B341 213 (1990); B. Brugmann and J. Pullin, Nucl. Phys. B390 399 (1993).

[4] E. T. Newman and C. Rovelli, Phys. Rev. Lett. 69, 1300 (1992).

[5] B. S. DeWitt, in Gravitation, an intorduction to current research, edited by . L. Witten (Wiley, New York, 1962).

[6] C. Rovelli, Class. and Quant. Gravity 8297 (1991).

[7] K. V. Kuchar and C. G. Torre, Phys. Rev. D 43419 (1991);

Phys. Rev. D 443116 (1991).

[8] V. Husain, General covariance, loops, and matter, gr-qc 9304010, to appear in Phys. Rev. D. (1993).

[9] C. Rovelli, A generally covariant quantum field theory, University of Pittsburgh preprint (1992).

[10] L. Smolin, Diffeomorphism invariant observables in quantum gravity from a dynamical theory of surfaces, Syracuse University preprint (1992); Time, measurement and information loss in quantum cosmology, in Directions in General Relativity Vol 1, edited by. B.L. Hu and T. A. Jacobson, (Cambridge University Press, Cambridge 1992). 
[11] A. Ashtekar, C. Rovelli, and L. Smolin, Phys. Rev. Lett. 69, 237 (1992). 